\title{
On an Extensible Rule-based Prover for Event-B
}

\author{
Issam Maamria, Michael Butler, Andrew Edmunds, and Abdolbaghi Rezazadeh \\ ECS, University of Southampton, Southampton SO17 1BJ, UK \\ \{im06r, mjb, ae2, ra3\}@ecs.soton.ac.uk
}

\section{Motivation}

The Rodin platform [3] provides the practical setting to carry out modelling in Event-B. It seamlessly integrates modelling and proving, and provides an extensible and configurable mechanism that can be adapted to different application domains and development methods [1]. The Rodin platform provides a proving infrastructure that has certain limitations. Extending the prover with proof rules (rewrite and inference rules) requires a certain level of competence using the Java programming language as well as good knowledge of the toolset's internal architecture. A further complication of this approach is that it became non-trivial to verify the soundness of the prover after adding new rules. This paper presents our approach when dealing with prover extensibility and soundness in the context of Event-B.

\section{The Theory Construct}

Theories will provide a mechanism by which the user can extend the proof capabilities of the Rodin platform by specifying rewrite rules. Proof obligations will be generated to verify the soundness of the prover augmented with the new rules. In essence, the theory construct will allow a degree of meta-reasoning to be carried out within the same platform in a similar fashion to Event-B reasoning. Figure 1 outlines the structure of the theory construct. In what follows, we briefly describe each of the elements of the theory construct:

1. Sets. A theory can define a number of given sets on which it is parametrised.

2. Metavariables. A theory can define a number of metavariables each of which has a type.

3. Rewrite Rules. Rewrite rules are one-directional equations that can be used to rewrite formulas to equivalent forms. As part of specifying a rewrite rule, the theory developer decides whether the rule can be applied automatically without user intervention or interactively following a user request.

\section{Rewrite Rules}

A rewrite rule defines how a formula lhs may be rewritten to one of several formulae $r h s_{i}$ provided condition $C_{i}$ holds. The following two definitions formalise the concept of rewrite rules within theories. Note the use of the well-definedness operator $\mathcal{D}[2]$. 


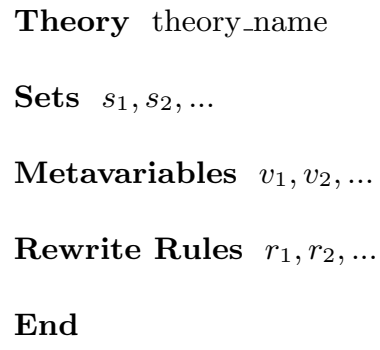

Fig. 1. The Theory Construct

Definition 1 (Rewrite Rule). A rewrite rule is of the form

$$
\begin{aligned}
& l h s \rightarrow C_{1}: r h s_{i} \\
& \ldots \\
& C_{n}: r h s_{n}
\end{aligned}
$$

where:

1. $n \geq 1$,

2. Ihs is not a meta-variable but may contain metavariables,

3. lhs and $r h s_{i}$ (for all $i$ such that $1 \leq i \leq n$ ) are formulas of the same syntactic class i.e., both expressions or both predicates,

4. $C_{i}$ (for all $i$ such that $1 \leq i \leq n$ ) are predicates,

5. $C_{i}$ and $r h s_{i}$ (for all $i$ such that $1 \leq i \leq n$ ) only contain free variables from lhs,

6. lhs and $r h s_{i}$ (for all $i$ such that $1 \leq i \leq n$ ) have the same type if lhs is an expression.

Note. In this paper, we only consider rewrite rules whose left hand side is a basic predicate (e.g., $\subseteq$ ) or is an expression not involving binding. More generally, we do not consider rules that require side conditions (i.e., non-freeness conditions).

Definition 2 (Sound Rewrite Rule). A rewrite rule

$$
\begin{aligned}
l h s \rightarrow C_{1} & : r h s_{i} \\
& \ldots \\
C_{n} & : r h s_{n}
\end{aligned}
$$

is said to be sound if the following sequents are valid:

1. $H, \mathcal{D}($ lhs $) \vdash \mathcal{D}\left(C_{i}\right)$ for all $i$ such that $1 \leq i \leq n$,

2. $H, \mathcal{D}($ lhs $), C_{i} \vdash \mathcal{D}\left(r h s_{i}\right)$ for all $i$ such that $1 \leq i \leq n$, 
3. (a) $H, \mathcal{D}($ lhs $), C_{i} \vdash$ lhs $=$ rhs $_{i}$ for all $i$ such that $1 \leq i \leq n$ if lhs is an expression, or;

(b) $H, \mathcal{D}($ lhs $), C_{i} \vdash$ lhs $\Leftrightarrow r h s_{i}$ for all $i$ such that $1 \leq i \leq n$ if lhs is a predicate,

where $H$ is a predicate providing typing information for all free variables occurring in lhs.

The previous definition ensures that rewrite rules are both validity-preserving and WD-preserving.

\section{The Theory Prototype Plug-in}

A theory prototype plug-in has been developed as an extension to the Rodin platform. The plug-in offers the following capabilities:

1. Users can develop and validate theories in the same way as contexts and machines.

2. Users can deploy theories to a specific directory where they become available to the interactive and automatic provers of Rodin.

3. Users can use rewrite rules defined within the deployed theories as a part of the proving activity. A pattern matching mechanism is implemented to calculate applicable rewrite rules to any given sequent.

\section{Further Work}

This work can be extended to enable the specification and validation of inference rules within theories. It can also be extended by providing a clear set of guidelines to help the theory developer with deciding whether a rule can be applied automatically. Finally, the verification of the pattern matching mechanism will give more confidence in this approach.

\section{References}

1. J.-R. Abrial, M. Butler, S. Hallerstede, and L. Voisin. An open extensible tool environment for Event-B. In International Conference on Formal Engineering Methods (ICFEM), 2006.

2. Jean-Raymond Abrial and Louis Mussat. On using conditional definitions in formal theories. In $Z B$ '02: Proceedings of the 2nd International Conference of $B$ and $Z$ Users on Formal Specification and Development in $Z$ and B, pages 242-269, London, UK, 2002. Springer-Verlag.

3. Michael Butler and Stefan Hallerstede. The Rodin Formal Modelling Tool. BCSFACS Christmas 2007 Meeting - Formal Methods In Industry, London., December 2007. 